(2) Open Access Full Text Article

REVIEW

\title{
Human La Protein: An RNA-Binding Protein Involved in Ovarian Cancer Development and Multidrug Resistance
}

This article was published in the following Dove Press journal: OncoTargets and Therapy

\section{Xuan Huang \\ Jing Tang (D)}

Department of Pharmacy, Obstetrics \& Gynecology Hospital of Fudan University, Shanghai 2000II, People's Republic of China
Correspondence: Jing Tang

Department of Pharmacy, Obstetrics \&

Gynecology Hospital of Fudan University,

Shanghai 2000II, People's Republic of

China

Email 18I7@fckyy.org.cn

\begin{abstract}
Multidrug resistance is the main cause of chemotherapy failure and death in patients with advanced ovarian cancer. Drug resistance is a problem that must be solved to improve the survival rate of patients with advanced ovarian cancer. The RNA-binding protein $\mathrm{La}$ and the La-related protein family are highly expressed in various malignant tumors, including ovarian cancer. This article reviews the mechanisms of La protein in tumorigenesis, development, and drug resistance. High La protein expression in tumor cells promotes tumor proliferation, invasion, and migration; disrupts cell cycle; and inhibits tumor cell apoptosis caused by chemotherapeutic drugs through various pathways, resulting in chemotherapy resistance in ovarian cancer. Further study of the role of La protein in ovarian cancer multidrug resistance may be conducive to the development of human La protein-specific inhibitors that suppress ovarian cancer drug resistance.
\end{abstract}

Keywords: La protein, Sjögren's syndrome antigen B, ovarian cancer, chemotherapy drug resistance, multidrug resistance, ovarian cancer stem cells

\section{Introduction}

Ovarian cancer is the most common cause of gynecological cancer-related death. Approximately 240,000 new cases of ovarian cancer are diagnosed each year, leading to 140,000 deaths from the disease. ${ }^{1}$ At present, surgery and platinumbased chemotherapy are the main treatments for ovarian cancer. In the past 40 years, advancements in surgery and chemotherapy have only slightly improved ovarian cancer treatment outcomes. For example, in the United States, the 5-year survival rate for ovarian cancer has increased from $40 \%$ in 1985 to $45 \%$ in 2015 (the 5-year survival rate for breast cancer in the same year was $90 \%$ ). ${ }^{2}$ Ovarian cancer treatment resistance is one of the main reasons for its high mortality. ${ }^{3}$ Eighty percent of women respond well to initial treatment with platinum drugs, but almost all patients will experience multiple disease relapses, and the diseasefree interval time becomes increasingly shorter, to the point that ovarian cancer will eventually become platinum-resistant, leading to the death of most ovarian cancer patients. ${ }^{4,5}$ Successful ovarian cancer treatment requires overcoming the resistance to standard platinum-based therapies, but the development of such resistance is almost inevitable.

In recent years, poly-ADP-ribose polymerase (PARP) inhibitors have been identified as new first-line and neoadjuvant chemotherapy drugs for treatment of 
ovarian cancer that results from homologous recombination (HR) DNA repair pathway defects. Among them, olaparib, niraparib, and rucaparib have been approved for treatment of recurrent epithelial ovarian cancer. However, BRCA1/2 gene mutations and defects in other genes that affect DNA damage repair (such as $R A D 51 C, R A D 51 D, B I R P 1$, and PALB2) will lead to resistance to PARP inhibitors in ovarian cancer. ${ }^{6}$ Moreover, clinical studies have shown that the objective response rate of the PARP inhibitor olaparib in longterm ovarian cancer treatment is lower than expected, the therapeutic effect of PARP inhibitors is difficult to sustain over the long term, ${ }^{7}$ the response rate to further treatment after cancer progression is low, and there is cross-resistance to platinum, paclitaxel, and other chemotherapy drugs. ${ }^{8}$ This multidrug resistance (MDR) is the main reason chemotherapy fails in advanced ovarian cancer. ${ }^{9}$ A variety of mechanisms are related to MDR in ovarian cancer, including 1) changes in apoptosis, cell cycle and DNA damage and repair mechanisms; 2) the activation and maintenance of cancer stem cells (CSCs); 3) changes in the drug efflux system, drug metabolism, and drug target structure; and 4) changes in cell membrane composition and epithelial mesenchymal transition. ${ }^{10,11}$

The RNA-binding protein La and the La-related protein (LARP) family are highly expressed in various malignant tumors and are related to tumor cell proliferation, invasion, migration, and apoptosis; cell cycle changes; and the expression and maintenance of CSC-like traits in ovarian cancer. La protein and the LARP family may be involved in ovarian cancer occurrence and progression and the mechanism of MDR; thus, they might become targets of specific inhibitors to mitigate ovarian cancer progression and drug resistance.

\section{The Structure of La Protein}

La protein, also known as Sjögren's syndrome antigen $\mathrm{B}$ (SSB), is a multifunctional RNA-binding protein containing 408 amino acids that is conserved in almost all eukaryotes, playing important functions in RNA processing and metabolism. ${ }^{12}$ La protein binds RNA through its La motif (LAM), RNA recognition motif 1 (RRM1), and noncoding RRM2. RRM2 is located at the characteristic C-terminal extension of the mammalian La protein. The C-terminal region of La protein also contains a nuclear localization signal, nucleolar localization signal, nuclear retention signal, and RNA chaperone domain. Moreover, the LAM can be combined with other motifs to form other LARPs, such as LARP1. ${ }^{13}$ Domain organizations among La protein and LARP1 are shown in Figure 1. La protein primarily localizes to the nucleus and nucleolus and can also shuttle between the cytoplasm and nucleus. ${ }^{14}$ The relative molecular weight of La protein is $47 \mathrm{kD}$, and $\mathrm{La}$ is involved in all levels of RNA metabolism.

\section{Functions of La Protein}

La protein can specifically bind the terminal motif UUU$3^{\prime} \mathrm{OH}$ of RNA polymerase III transcripts to promote RNA biosynthesis, ${ }^{18,19}$ protecting them from exonuclease degradation. La protein promotes the production of tRNAs and processes the $5^{\prime}$ and $3^{\prime}$ ends of the pre-tRNA precursors to act as an RNA chaperone protein. La protein binds to specific types of RNA molecules to regulate their downstream processing, prevent misfolding, and promote the correct folding and maturation of transcripts. ${ }^{15,19,20}$ La protein is also involved in the translation process mediated by the internal ribosome entry site (IRES) in mRNAs. ${ }^{21,22}$ IRES-mediated protein translation is initiated during cellular stress and apoptosis. Under stress conditions, the translation of capped eukaryotic mRNAs is downregulated. However, IRES

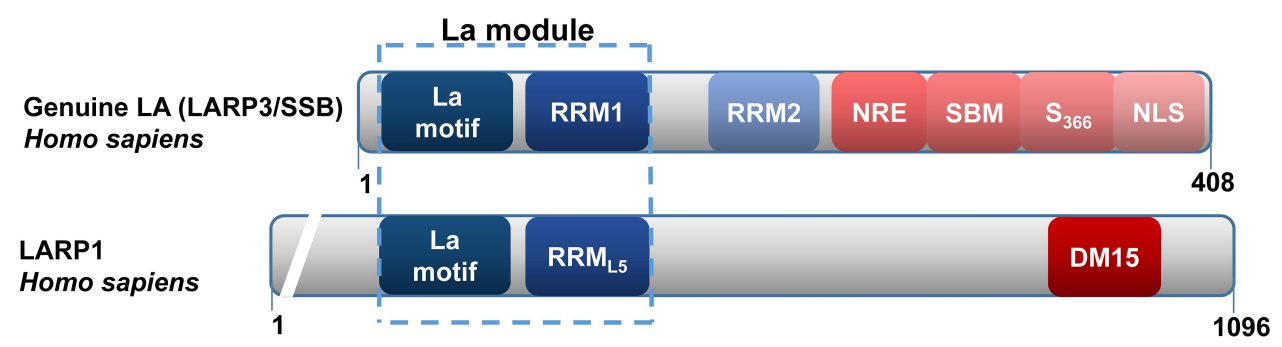

Figure I Domain organizations among La protein and La-related protein I (LARPI).

Abbreviations: LARPI, La-related protein I; RRM, RNA recognition motif; NRE, nuclear retention element; ${ }^{15}$ SBM, short basic motif for recognition of $5^{\prime}$ PPPG of nascent

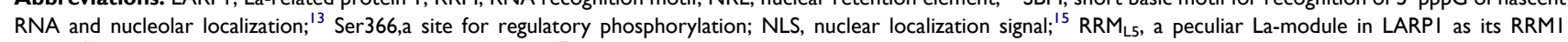
moiety; ${ }^{16}$ DMI5, a highly conserved C-terminus in LARPI. ${ }^{17}$ 
elements can offset this downregulatory mechanism by promoting translation, allowing survival of cells under stress conditions and mediating drug resistance of cancer cells. ${ }^{23}$

La protein and LARP1 are highly expressed in multiple malignant tumors, including ovarian cancer, and are related to the clinical tumor, node, metastasis (TNM) stage of the tumors, the presence of lymph node metastasis, and the degree of cancer cell differentiation. They play important roles in the translation regulation of cancer cell tumorpromoting factors. ${ }^{24-28}$ La protein can promote the tumorigenicity of cancer cells, disrupt cell cycle, and promote tumor cell proliferation, migration, and invasion. ${ }^{24}$ Moreover, high LARP1 expression predicts a poor prognosis in ovarian cancer patients. ${ }^{27}$ As shown in Figure 2, La protein plays a role in many aspects of the development of malignant tumors.

\section{La Protein and $\beta$-Catenin}

The expression of $\beta$-catenin depends on the level of cellular La protein. Elevated La protein content can directly stimulate the translation of $\beta$-catenin mRNA or stimulate the positive regulators of $\beta$-catenin expression. ${ }^{29} \beta$-Catenin plays an important role in the proliferation and metastasis of ovarian cancer cells. ${ }^{30} \beta$-Catenin is a component of the adhesion junction in the cell membrane that regulates cellcell adhesion and can also enter the nucleus after Wnt signal activation and upregulate tumor-promoting genes. ${ }^{31} \mathrm{Wnt} / \beta$ - catenin signaling is significantly associated with ovarian cancer occurrence, tumor growth, tumor metastasis, tumor dormancy, tumor immunity, and maintenance of CSC traits. ${ }^{32}$ The Wnt/ $\beta$-catenin signaling pathway upregulates the expression of cell cycle-related proteins, such as cyclin B1 and cyclin C, to promote the occurrence and development of cancer. ${ }^{33}$ La protein may promote ovarian cancer cell proliferation, differentiation, survival, adhesion, migration, invasion, and tumor growth in nude mice through the $\mathrm{Wnt} / \beta$-catenin pathway. ${ }^{29}$

\section{La Protein and X-Linked Inhibitor of Apoptosis Protein (XIAP)}

XIAP is a member of the inhibitor of apoptosis (IAP) family of proteins and an inhibitor of endogenous and exogenous apoptosis. Overexpression of XIAP is intimately associated with the progression, recurrence, prognosis, tumor aggressiveness, and resistance to chemotherapy and targeted therapy in ovarian cancer. ${ }^{34}$ XIAP translation is controlled by the IRES element located $162 \mathrm{nt}$ upstream of the start site in the XIAP 5' untranslated region (UTR). IRES-mediated XIAP translation is crucial to the role of XIAP in apoptosis. La protein is an essential component of the XIAP IRES ribonucleoprotein complex. La protein specifically

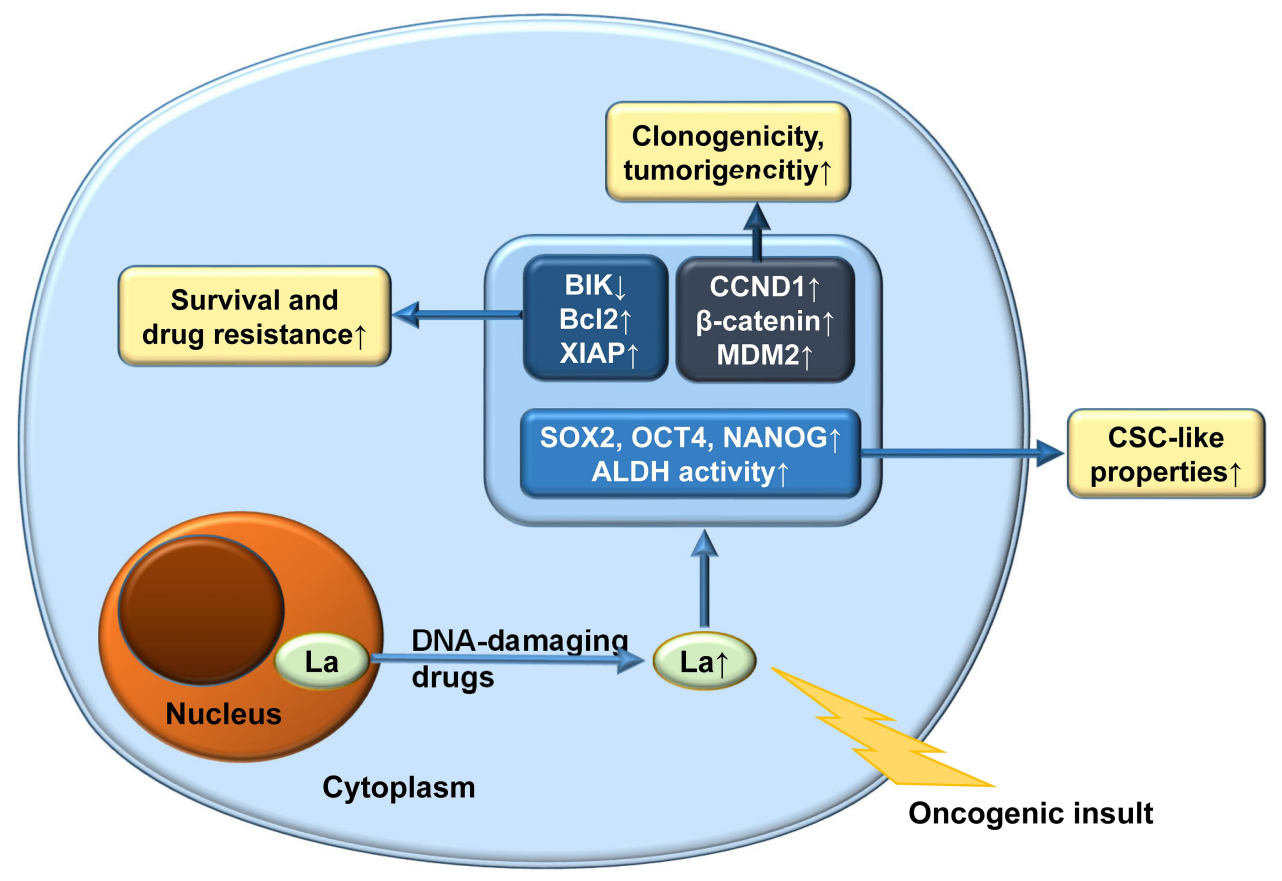

Figure 2 Roles of La protein as a tumor promoter and multidrug resistance agent.

Abbreviations: $\mathrm{Bcl}$ 2, B-cell lymphoma 2; BIK, Bcl2 interacting killer; XIAP, X-linked inhibitor of apoptosis protein; CCNDI, cyclin DI; MDM2, murine double minute 2; $\mathrm{ALDH}$, aldehyde dehydrogenase; CSC, cancer stem cell. 
binds to the XIAP IRES sequence and interacts with XIAP IRES to form the core of the ribonucleoprotein complex. La protein enhances IRES-dependent translation of the apoptosis inhibitor XIAP to upregulate its expression. ${ }^{35}$

\section{La Protein and Murine Double Minute 2 (MDM2)}

La protein activates translation of MDM2 mRNA by binding to the $5^{\prime}$ UTR of MDM2 mRNA. ${ }^{36}$ MDM2 is highly expressed in ovarian cancer cells. MDM2 can bind to the $\mathrm{N}$-terminal domain of p53, inhibit TP53 gene transcription, and amplify itself without regulation, eventually leading to the occurrence of malignant tumors. ${ }^{37}$ p53 is a tumor suppressor involved in various cellular mechanisms, including DNA repair, apoptosis, and cell cycle arrest. The p53 protein expression level is controlled by MDM2. p53 prevents tumor growth in ovarian cancer progression, and thus, any loss of p53 function will contribute to the development of ovarian cancer. Type II ovarian cancer is a highly aggressive type of ovarian cancer and includes advanced serous carcinoma, undifferentiated carcinoma, and carcinosarcoma. Almost all of these ovarian cancer types have TP53 mutations and genomic instability. ${ }^{3}$

\section{La Protein and GI/S-Specific Cyclin DI (CCNDI)}

Abnormally overexpressed La protein stimulates IRESmediated translation of G1/S-specific CCND1 mRNA by regulating the CCND1 gene. ${ }^{38} \mathrm{CCND} 1$ regulates cell cycle progression from G1/S phase. Overexpression of CCND1 may significantly shorten the G1-S phase interval and accelerate cell proliferation, even causing it to speed out of control. ${ }^{39}$ La protein is necessary for cell proliferation, and depletion of La protein at any cell cycle stage will cause cell cycle arrest. La protein binds to CCND1 mRNA and acts as a molecular chaperone, allowing the internal ribosomes to enter the IRES translation process and thereby increasing CCND1 expression, disrupting cell cycle progression, and eventually affecting cell proliferation. ${ }^{40}$ Overexpression of CCND1 has been correlated with a shortened survival time of cancer patients and increased cancer cell metastasis in ovarian cancer. ${ }^{39}$ Silencing CCND1 will damage DNA double-strand break repair, weaken olaparib resistance in ovarian cancer, and sensitize $B R C A 1$-wild-type ovarian cancer cells to olaparib. ${ }^{41}$

\section{Anti-Apoptotic Effect of La Protein}

La protein can promote expression of the pro-apoptotic protein B-cell lymphoma 2 (Bcl2), ${ }^{42}$ and this overexpression helps tumor cells develop resistance to cisplatininduced apoptosis. La protein tightly binds to the translation initiation site of Bcl2 and unwinds and embeds itself into the secondary structure of the translation initiation site through its chaperone activity to stimulate $B c l 25^{\prime}$ UTRdriven expression. Bcl2 is an important anti-apoptotic oncoprotein that promotes chemotherapy resistance in ovarian cancer and is associated with patient prognosis. Low $\mathrm{La}$ protein expression is associated with low $\mathrm{Bcl} 2$ protein expression and high cisplatin sensitivity and can increase the sensitivity of tumor cells to cisplatin therapy. La can stimulate $\mathrm{Bcl} 2$ mRNA translation and promote cisplatin resistance during cisplatin treatment. Moreover, the expression of XIAP is regulated by La protein, ${ }^{35}$ and thus, La depletion will simultaneously reduce Bcl2 and XIAP expression. ${ }^{42}$ Inhibition of XIAP can promote carboplatininduced apoptosis of ES2 and 3AO ovarian cancer cells, thereby reducing their carboplatin resistance. ${ }^{43}$

An increase in La protein expression can directly stimulate upregulation of $\beta$-catenin, ${ }^{29}$ and $\mathrm{La}$ protein is a substrate of Akt. ${ }^{18} \mathrm{Wnt} / \beta$-catenin and the phosphatidylinositol 3-kinase (PI3K)/Akt pathway are important in ovarian cancer development. These two pathways are interconnected at multiple levels and involve multiple upstream and downstream common effectors. Glycogen synthase kinase $3 \beta$ (GSK-3 $\beta$ ) plays an important role in triggering $\beta$-catenin instability. PI3K/Akt signaling can regulate the $\beta$-catenin level by influencing GSK-3 $\beta$ activity. ${ }^{44-46}$ In turn, Wnt/ $\beta$-catenin signaling can affect the PI3K/Akt signaling pathway through the Wnt receptor Frizzled. ${ }^{47}$ These findings have led to the hypothesis that the Wnt/ $\beta$-catenin and PI3K/Akt pathways may be combined therapy targets of ovarian cancer inhibitors. ${ }^{48}$ Oncogenic mutations in the PI3K/Akt pathway are among the most common changes in cancer. ${ }^{49}$ Activation of the PI3K/Akt-NF-kB-IAP pathway inhibits the apoptosis of ovarian cancer cells induced by cisplatin and programmed cell death protein 1 (PD-1) inhibitor, resulting in cisplatin and PD-1 inhibitor drug resistance in ovarian cancer. ${ }^{50}$ In one study on 655 ovarian cancer patients, the average PI3K, Akt, and NF-kB1 mRNA expression levels were increased, and the high expression level of this pathway was associated with a lower overall survival rate. ${ }^{51}$ We speculate that La protein may lead to 
inhibition of chemotherapy drug-induced apoptosis of ovarian cancer cells through $\mathrm{Wnt} / \beta$-catenin and $\mathrm{PI} 3 \mathrm{~K} /$ Akt-NF-kB-IAP signaling.

Like La protein, LARP1 is associated with ovarian cancer cell apoptosis through a similar mRNA translation regulatory mechanism. After LARP1 knockdown, the mRNA levels of survival genes, such as Bcl2, Erbb3, and $A k t 3$, are significantly reduced, while the expression of proapoptotic genes, including Bik (Bcl2 interacting killer), Tnf (tumor necrosis factor), and Dapk2 (death associated protein kinase 2), are increased. LARP1 is a component of the $\mathrm{Bcl} 2$ messenger ribonucleoprotein (mRNP) complex, and its DM15 domain binds to the $B c l 23^{\prime}$ UTR to enhance the stability of $B c l 2$ transcripts. LARP1 can also form an mRNP with $B i k$, which encodes a pro-apoptotic protein. LARP1 exerts a binary effect on $B c l 2$ and $B i k$, stabilizing $B c l 2$ and at the same time destabilizing Bik mRNA, with a net antiapoptosis effect. Since LARP1 acts as a positive and negative posttranscriptional regulator of the apoptosis regulators $B c l 2$ and $B i k$, respectively, regulation of LARP1 expression in ovarian cancer cells will affect the survival and platinum resistance of these cells. Knockout of LARP1 can increase the cisplatin-caused apoptosis of ovarian cancer cells 4-fold, thereby significantly improving the platinum resistance of ovarian cancer cells. ${ }^{25}$

\section{La Protein and CSC-Like Population in Ovarian Cancer}

Ovarian cancer occurrence and development are closely related to CSCs. Prominent features of CSCs include selfrenewal, immortality, pluripotency, the ability to differentiate into different tumor cell subtypes, and the ability to regulate and balance their self-renewal and differentiation in response to environmental and nonenvironmental stimuli, all of which contribute to the occurrence, progression, drug resistance, and metastasis of primary ovarian cancer. ${ }^{11} \mathrm{CSCs}$ are usually partially resistant to standard treatments, which is also an important cause of ovarian cancer drug resistance. CSCs are identified and characterized by specific cell-surface and intracellular markers. Increased activity of aldehyde dehydrogenase (ALDH); increased abundance of certain cell populations, such as $\mathrm{CD} 133^{+}$cells $;{ }^{52}$ and high expression of certain important embryonic stem cell-related transcription factors (such as SOX2, OCT4, NANOG, and c-MYC, which are involved in ovarian cancer progression and drug resistance ${ }^{53,54}$ ) are associated with an enhanced CSC-like trait in ovarian cancer. ALDH, CD133, SOX2, OCT4, and NANOG can be used to identify ovarian cancer CSCs. ${ }^{11}$ Knockdown of LARP1 results in decreased ALDH activity, a decreased $\mathrm{CD} 133^{+}$cell population size, and decreased NANOG, SOX2, and OCT4 expression in OVCAR3 ovarian cancer cells. LARP1 promotes several characteristics associated with CSC-like cells, namely, clonal formation, carcinogenicity, chemotherapy resistance, and stem cell-related gene expression. ${ }^{27}$

\section{Conclusion}

Solving the problem of the high drug resistance rate in ovarian cancer is urgently needed to reduce the mortality rate of women. La protein and the LARP family may promote ovarian cancer cell proliferation, invasion, and migration by promoting $\beta$-catenin, MMP-2, and CCND1 expression; inhibit ovarian cancer drug resistance by promoting $\mathrm{Bcl} 2$ and XIAP expression; and inhibit ovarian cancer cell apoptosis and participate in ovarian cancer MDR by participating in $\mathrm{Wnt} / \beta$ catenin and PI3K/Akt signaling. LARP1 promotes ovarian cancer progression and drug resistance by maintaining CSClike traits in ovarian cancer. La protein is not only involved in platinum resistance but may also contribute to resistance to PARP inhibitors and PD-1 inhibitors by inhibiting apoptosis and the PI3K/Akt pathway. Current research on the inhibition of tumor drug resistance by La protein mainly focuses on platinum-based chemotherapeutic drugs. We speculate that La protein may participate in the processes of drug resistance development in response to many anti-tumor drugs through similar anti-apoptotic pathways. La protein is an extremely promising target for solving the problem of drug resistance in ovarian cancer. We believe that more research on the mechanism of La protein in ovarian cancer drug resistance will provide a basis for the development of specific La protein inhibitors and that development of these specific inhibitors may open up new ways to overcome MDR in ovarian cancer. Combined administration of $\mathrm{La}$ protein inhibitors may improve the drug resistance caused by long-term application of platinum drugs, PARP inhibitors, and PD-1 inhibitors and reduce the needed doses of these drugs. These effects will help alleviate the pain of chemotherapy, improve the efficacy of chemotherapy, and reduce the chemotherapy cost to ovarian cancer patients. Thus, the development of specific La protein inhibitors may directly improve the treatment experience of ovarian cancer patients and clinical outcomes.

\section{Acknowledgments}

This study was funded by a grant from Shanghai Key Specialty Project of Clinical Pharmacy (no. AB83110 002017005) 


\section{Author Contributions}

Xuan $\mathrm{H}$ wrote the manuscript and collected the data; Jing Tang conceived the idea, supervised the writing, revised the manuscript and directed all progress. All authors contributed to data analysis, drafting or revising the article, have agreed on the journal to which the article will be submitted, gave final approval of the version to be published, and agree to be accountable for all aspects of the work.

\section{Authors Declaration and Warranties}

This article is original, and does not infringe any existing copyright or any other rights prescribed by law. To the best of our knowledge all statements contained in it purporting to be factual are true and correct.

\section{Disclosure}

The authors declare no conflicts of interest for this work.

\section{References}

1. Webb PM, Jordan SJ. Epidemiology of Epithelial Ovarian Cancer. Best Pract Res Clin Obstet Gynaecol. 2017;41:3-14.

2. Holmes D. Ovarian cancer: beyond resistance. Nature. 2015;527 (7579):217.

3. Lupia M, Cavallaro U. Ovarian cancer stem cells: still an elusive entity? Mol Cancer 2017;16(1):64.

4. Moore KN, Martin LP, Omalley DM, et al. A review of mirvetuximab soravtansine in the treatment of platinum-resistant ovarian cancer. Future Oncol. 2018;14(2):123-136.

5. Oronsky B, Ray CM, Spira AI, Trepel JB, Carter CA, Cottrill HM. A brief review of the management of platinum-resistant-platinumrefractory ovarian cancer. J Med Oncol. 2017;34(6):103.

6. Lorusso D, Tripodi E, Maltese G, et al. Spotlight on olaparib in the treatment of BRCA-mutated ovarian cancer: design, development and place in therapy. Drug Des Devel Ther. 2018;12:1501-1509.

7. Wakefield MJ, Nesic K, Kondrashova O, Scott CL. Diverse mechanisms of PARP inhibitor resistance in ovarian cancer. Biochim Biophys Acta Rev Cancer. 2019;1872(2):188307.

8. Cecere SC, Giannone G, Salutari V, et al. Olaparib as maintenance therapy in patients with BRCA 1-2 mutated recurrent platinum sensitive ovarian cancer: real world data and post progression outcome. Gynecol Oncol. 2020;156(1):38-44.

9. Gao B, Yang F, Chen W, et al. Multidrug resistance affects the prognosis of primary epithelial ovarian cancer. Oncol Lett. 2019;18 (4):4262-4269.

10. Khan IU, Khan RU, Asif H, et al. Co-delivery strategies to overcome multidrug resistance in ovarian cancer. Int J Pharm. 2017;533 (1):111-124.

11. Mihanfar A, Attari JA, Mohebbi I, et al. Ovarian cancer stem cell: a potential therapeutic target for overcoming multidrug resistance. J Cell Physiol. 2019;234(4):3238-3253.

12. Xiu X. Advance in molecular biology of lupus la protein. 2009.

13. Maraia RJ, Mattijssen S, Cruz-Gallardo I, Conte MR. The La and related RNA-binding proteins (LARPs): structures, functions, and evolving perspectives. Wiley Interdiscip Rev RNA. 2017;8(6):e1430.
14. Marrella SA, Brown KA, Mansourinoori F, Porat J, Wilson DJ, Bayfield MA. An interdomain bridge influences RNA binding of the human La protein. J Biol Chem. 2019;294(5):1529-1540.

15. Alfano C, Sanfelice D, Babon J, et al. Structural analysis of cooperative RNA binding by the La motif and central RRM domain of human La protein. Nat Struct Mol Biol. 2004;11(4):323-329. doi:10.1038/nsmb747

16. Fonseca BD, Lahr RM, Damgaard CK, Alain T, Berman AJ. LARP1 on TOP of ribosome production. Wiley Interdiscip Rev RNA. 2018;9 (5): 1480

17. Deragon JM, Bousquet-Antonelli C. The role of LARP1 in translation and beyond. Wiley Interdiscip Rev RNA. 2015;6(4):399-417. doi:10.1002/wrna. 1282

18. Teplova M, Yuan YR, Phan AT, et al. Structural basis for recognition and sequestration of $\mathrm{UUU}(\mathrm{OH}) 3^{\prime}$ temini of nascent RNA polymerase III transcripts by La, a rheumatic disease autoantigen. Mol Cell. 2006;21(1):75-85. doi:10.1016/j.molcel.2005.10.027

19. Intine RVA, Tenenbaum SA, Sakulich AL, Keene JD, Maraia R. Differential phosphorylation and subcellular localization of $\mathrm{La}$ RNPs Associated with precursor tRNAs and translation-related mRNAs. Mol Cell. 2003;12(5):1301-1307.

20. Gourzi VC, Kapsogeorgou EK, Kyriakidis NC, Tzioufas AGJC, Immunology E. Study of microRNAs (miRNAs) that are predicted to target the autoantigens Ro/SSA and La/SSB in primary Sjögren's Syndrome. Clin Exp Immunol. 2015;182(1):14-22.

21. Costamattioli M, Svitkin YV, Sonenberg N, Biology C. La autoantigen is necessary for optimal function of the poliovirus and hepatitis $\mathrm{C}$ virus internal ribosome entry site in vivo and in vitro. $J \mathrm{Mol} \mathrm{Cell}$ Biol. 2004;24(15):6861-6870.

22. Pudi R, Srinivasan P, Das S. La protein binding at the GCAC site near the initiator AUG facilitates the ribosomal assembly on the hepatitis C virus RNA to influence internal ribosome entry site-mediated translation. J Biol Chem. 2004;279(29):29879-29888.

23. Gao W, Li Q, Zhu R, Jin JJ. La autoantigen induces Ribosome Binding Protein 1 (RRBP1) Expression through Internal Ribosome Entry Site (IRES)-mediated translation during cellular stress condition. Int J Mol Sci. 2016;17(7):1174.

24. Chen K, Wu Y, Li M, Li L, Gao Y, Gao QJ. La protein expression in cervical cancer tissues and its clinical significance. Int $J$ Mol Sci. 2013;33(7):999.

25. Hopkins TG, Mura M, Alashtal HA, et al. The RNA-binding protein LARP1 is a post-transcriptional regulator of survival and tumorigenesis in ovarian cancer. Nucleic Acids Res. 2016;44(3):1227-1246.

26. Debao F, Dake H, Fei J. [The role of La-related protein 1 in the progression of gallbladder cancer]. Acta Univ Med Anhui. 2020;55 (02):282-286. Chinese.

27. Liu X, Huang Z, Guo X, et al. Role of la-related protein 1 expression in gastric carcinoma. $J$ Pract Med. 2017;33(07):1116-1119.

28. Zhang D. [Screening of La Protein Interacting Proteins in Human Breast Cancer Cells by Immunocoprecipitation and Mass Spectrometry]. The 12th Annual Meeting of the Chinese Society of Biotechnology; 2018; Changsha, China: Cnki: 2018. Chinese.

29. Sommer G, Rossa C, Chi AC, Neville BW, Heise TJPO. Implication of RNA-binding protein la in proliferation, migration and invasion of lymph node-metastasized hypopharyngeal SCC cells. PloS One. 2011;6(10):8.

30. Wang H, Makki MS, Wen J, et al. Overexpression of $\beta$-catenin and cyclinD1 predicts a poor prognosis in ovarian serous carcinomas. Int J Clin Exp Pathol. 2014;7(1):264-271.

31. Harris TJC, Tepass U. Adherens junctions: from molecules to morphogenesis. Nat Rev Mol Cell Biol. 2010;11(7):502-514.

32. Shang S, Hua F, Hu Z. The regulation of $\beta$-catenin activity and function in cancer: therapeutic opportunities. Oncotarget. 2017;8 (20):33972-33989.

33. Vilchez V, Turcios L, Marti F, Gedaly R. Targeting Wnt/B-catenin pathway in hepatocellular carcinoma treatment. World J Gastroenterol. 2016;22(2):823-832. 
34. Evans MK, Sauer SJ, Nath S, et al. X-linked inhibitor of apoptosis protein mediates tumor cell resistance to antibody-dependent cellular cytotoxicity. Cell Death Dis. 2016;7(1):e2073-e2073.

35. Holcik M, Korneluk RGJM, Biology C. Functional characterization of the X-Linked Inhibitor of Apoptosis (XIAP) Internal ribosome entry site element: role of la autoantigen in XIAP translation. Mol Cell Biol. 2000;20(13):4648-4657.

36. Trotta R, Vignudelli T, Candini $\mathrm{O}$, et al. BCR/ABL activates mdm2 mRNA translation via the La antigen. Cancer Cell. 2003;3 (2):145-160.

37. Dogan E, Saygili U, Tuna B, et al. p53 and mdm2 as prognostic indicators in patients with epithelial ovarian cancer: a multivariate analysis. Gynecol Oncol. 2005;97(1):46-52.

38. Kuehnert J, Sommer G, Zierk AW, et al. Novel RNA chaperone domain of RNA-binding protein $\mathrm{La}$ is regulated by AKT phosphorylation. Nucleic Acids Res. 2015;43(1):581-594. doi:10.1093/nar/gku1309

39. Dhar KK, Branigan K, Parkes J, et al. Expression and subcellular localization of cyclin D1 protein in epithelial ovarian tumour cells. $\mathrm{Br}$ J Cancer. 1999;81(7):1174-1181.

40. Sommer G, Dittmann J, Kuehnert J, et al. The RNA-binding protein La contributes to cell proliferation and CCND1 expression. Oncogene. 2011;30(4):434-444.

41. Zhong Q, Hu Z, Li Q, Yi T, Li J, Yang HJGO. Cyclin D1 silencing impairs DNA double strand break repair, sensitizes BRCA1 wildtype ovarian cancer cells to olaparib. Gynecol Oncol. 2019;152 (1):157-165.

42. Heise $\mathrm{T}$, Kota V, Brock A, et al. The La protein counteracts cisplatin-induced cell death by stimulating protein synthesis of anti-apoptotic factor Bcl2. Oncotarget. 2016;7(20):29664-29676.

43. Zhang Y, Huang F, Luo Q, et al. Inhibition of XIAP increases carboplatin sensitivity in ovarian cancer. Onco Targets Ther. 2018;11:8751-8759.

44. Wei A, Fan B, Zhao Y, et al. ST6Gal-I overexpression facilitates prostate cancer progression via the $\mathrm{PI} 3 \mathrm{~K} / \mathrm{Akt} / \mathrm{GSK}-3 \beta / \beta$-catenin signaling pathway. Oncotarget. 2016;7(40):65374-65388.
45. Zhu J, Hu H, Wang J, Yang Y, Yi P. PRR11 overexpression facilitates ovarian carcinoma cell proliferation, migration, and invasion through activation of the PI3K/AKT/ $\beta$-catenin pathway. Cell Physiol Biochem. 2018;49(2):696-705.

46. Zhang Q, Zhou L, Guan Y, Cheng Y, Han X. BENC-511, a novel PI3K inhibitor, suppresses metastasis of non-small cell lung cancer cells by modulating $\beta$-catenin/ZEB1 regulatory loop. Chem Biol Interact. 2018;294:18-27.

47. Kang DW, Lee BH, Suh YA, et al. Phospholipase D1 inhibition linked to upregulation of ICAT blocks colorectal cancer growth hyperactivated by Wnt/ $\beta$-catenin and PI3K/Akt signaling. Clinical Cancer Res. 2017;23(23):7340-7350.

48. Prossomariti A, Piazzi G, Alquati C, Ricciardiello L. Are Wnt/ $\beta$ catenin and $\mathrm{PI} 3 \mathrm{~K} / \mathrm{AKT} / \mathrm{mTORC} 1$ distinct pathways in colorectal cancer? Cell Mol Gastroenterol Hepatol. 2020.

49. Arques O, Chicote I, Puig I, et al. Tankyrase inhibition blocks Wnt/ $\beta$ catenin pathway and reverts resistance to $\mathrm{PI} 3 \mathrm{~K}$ and AKT inhibitors in the treatment of colorectal cancer. Clin Cancer Res. 2016;22(3):644-656.

50. Lotsberg ML, Wnuk-Lipinska K, Terry S, et al. AXL targeting abrogates autophagic flux and induces immunogenic cell death in drug-resistant cancer cells. J Thorac Oncol. 2020;15(6):973-999. doi:10.1016/j.jtho.2020.01.015

51. Rada M, Nallanthighal S, Cha J, et al. Inhibitor of apoptosis proteins (IAPs) mediate collagen type XI alpha 1-driven cisplatin resistance in ovarian cancer. Oncogene. 2018;37(35):4809-4820.

52. Parte S, Batra SK, Kakar SS. Characterization of stem cell and cancer stem cell populations in ovary and ovarian tumors. J Ovarian Res. 2018;11(1):69.

53. Suster NK, Grazio SF, Virantklun I, Verdenik I, Smrkolj S. Cancer stem cell-related marker NANOG expression in ovarian serous tumors: a Clinicopathological Study of 159 cases. Int J Gynecol Cancer. 2017;27(9):2006-2013.

54. Parte S, Smolenkov A, Batra SK, Ratajczak MZ, Kakar SS. Ovarian cancer stem cells: unraveling a germline connection. Stem Cells Dev. 2017;26(24):1781-1803.
OncoTargets and Therapy

\section{Publish your work in this journal}

OncoTargets and Therapy is an international, peer-reviewed, open access journal focusing on the pathological basis of all cancers, potential targets for therapy and treatment protocols employed to improve the management of cancer patients. The journal also focuses on the impact of management programs and new therapeutic agents and protocols on patient perspectives such as quality of life, adherence and satisfaction. The manuscript management system is completely online and includes a very quick and fair peer-review system, which is all easy to use. Visit http://www.dovepress.com/ testimonials.php to read real quotes from published authors. 